\title{
Correction to: Gene expression analyses on multi-target mode of action of black cohosh in menopausal complaints - a pilot study in rodents
}

\author{
Petra Stute $^{1}$ (D) Stefan Ehrentraut ${ }^{2} \cdot$ Hans-Heinrich Henneicke-von Zepelin ${ }^{3}$ D $\cdot$ Petra Nicken $^{3}$
}

Published online: 10 August 2021

(c) Springer-Verlag GmbH Germany, part of Springer Nature 2021

\section{Correction to: Archives of Gynecology and Obstetrics https://doi.org/10.1007/s00404-021-06105-8}

In the original article published, the table legends were erroneously incompletely transferred from the manuscript to the

Table 1 Number of differentially regulated genes

\begin{tabular}{lllll}
\hline Hi Hy & PRAE & OVX & OVX + iCR & Compensation \\
\hline PRAE & & 836 & 2401 & 213 \\
OVX & 943 & & 2210 & \\
OVX + iCR & 1760 & 1759 & & \\
Compensation & 349 & & & \\
\hline
\end{tabular}

Selection, filtering, and compensation analysis of differentially regulated genes within the hippocampus and hypothalamus. The data are shown as a cross tabulation with the top right side showing the data for the hippocampus (Hi) and the bottom left side showing the data for the hypothalamus (Hy). The compensation analysis identified features in which treatment with black cohosh counteracted the effect of ovariectomy. published version. The correct table (Tables 1, 2, 3, 4) legends are given below.

Apart from this many typographical errors have been corrected.

The original article has been corrected.

The original articles can be found online at https://doi.org/10. 1007/s00404-021-06105-8.

Petra Stute

petra.stute@insel.ch

1 Department of Obstetrics, Gynaecologic Endocrinology and Reproductive Medicine, University Women's Hospital, University Clinic of Bern, Friedbuehlstrasse 19, 3010 Bern, Switzerland

2 Arrows Biomedical Deutschland GmbH, Münster, Germany

3 Department of Regulatory Affairs, Schaper and Brümmer GmbH and Co. KG, Salzgitter, Germany 
Table 2 Selected target genes for qPCR

\begin{tabular}{|c|c|c|}
\hline No & Gene & Name \\
\hline \multicolumn{3}{|c|}{$\begin{array}{l}\text { 1. Target gene selection based on Table"GSEA compensation analysis " (FC }>1.5, p<0.2 \text {; Filter: compensation). Question: Which OVX- } \\
\text { induced changes are compensated by iCR? }\end{array}$} \\
\hline 1 & AVPR1A (Hi) & Arginine vasopressin receptor $1 \mathrm{~A}$ \\
\hline 2 & GAL (Hi) & Galanin and GMAP prepropeptide \\
\hline 3 & CALCA (Hi) & Calcitonin related polypeptide alpha \\
\hline 4 & HCRT (Hi) & Hypocretin neuropeptide precursor \\
\hline 5 & PNOC (Hi) & Prepronociceptin \\
\hline 6 & IL5 (Hi) & Interleukin 5 \\
\hline 7 & BCL2 (Hy) & BCL2, apoptosis regulator \\
\hline 8 & IGFBP1 (Hy) & Insulin like growth factor binding protein 1 \\
\hline 9 & IGFBP5 (Hy) & Insulin like growth factor binding protein 5 \\
\hline 10 & IL3 (Hy) & Interleukin 3 \\
\hline 11 & TRPV3 (Hy) & $\begin{array}{l}\text { Transient receptor potential cation channel } \\
\text { subfamily V member } 3\end{array}$ \\
\hline \multicolumn{3}{|c|}{$\begin{array}{l}\text { 2. Target gene selection based on "GSEA iCR exclusive " (FC }>1.5, p<0.2 \text {; Filter: iCR exclusive). Question: Which gene expression is exclu- } \\
\text { sively changed by iCR? }\end{array}$} \\
\hline 12 & PRL7A3 (Hi) & Prolactin family 7 , subfamily a, member 3 \\
\hline 13 & PLCB1 (Hy) & Phospholipase $\mathrm{C}$ beta 1 \\
\hline 14 & MAPK9 (Hy) & Mitogen-activated protein kinase 9 \\
\hline 15 & ADCY1 (Hy) & Adenylate cyclase 1 \\
\hline 16 & G6PC (Hy) & Glucose-6-phosphatase catalytic subunit \\
\hline 17 & CACNA1A (Hy) & Calcium voltage-gated channel subunit alpha1 A \\
\hline \multicolumn{3}{|c|}{$\begin{array}{l}\text { 3. Generally important target genes not covered in above mentioned lists. Question: Which further genes are of general importance in regard to } \\
\text { the present scientific work? }\end{array}$} \\
\hline 18 & ESR1 & Estrogen receptor 1 (alpha) \\
\hline 19 & ESR2 & Estrogen receptor 2 (beta) \\
\hline 20 & OPRM1 & Opioid receptor mu 1 \\
\hline 21 & KISS1 & KiSS-1 metastasis-suppressor \\
\hline 22 & TAC3 & Tachykinin 3 \\
\hline
\end{tabular}

Overview of selected target genes. $\mathrm{Hi}=$ Hippocampus, $\mathrm{Hy}=$ Hypothalamus, OVX = bilateral ovariectomy, iCR = isopropanolic extract of Cimicifuga racemosa (black cohosh). 


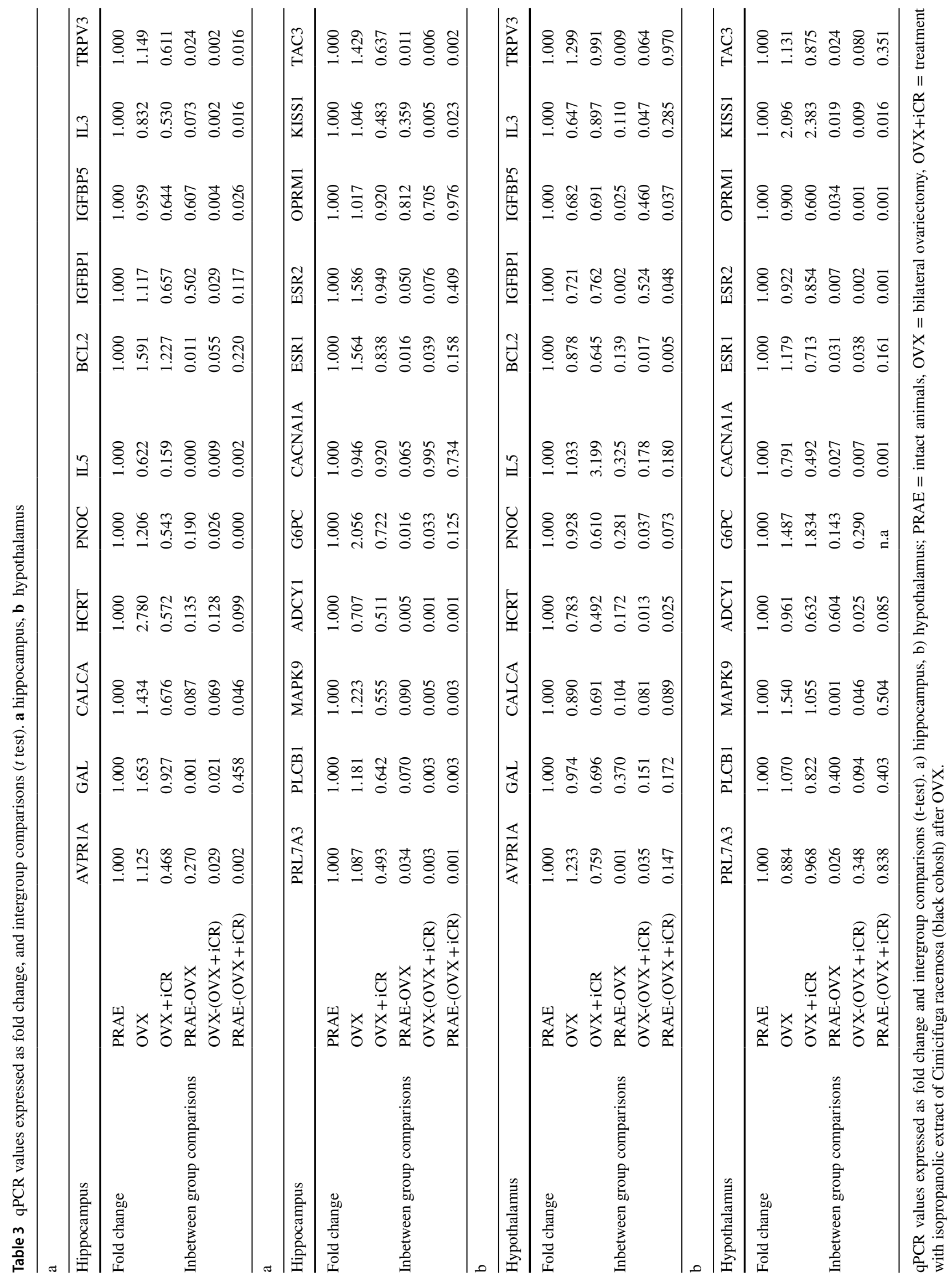


Table 4 Comparison of microarray and qPCR results

\begin{tabular}{|c|c|c|c|}
\hline Gene & Question for selecting this gene (details see text) & Hippocampus & Hypothalamus \\
\hline AVPR1A & Compensation (Hi) & $\#^{2}$ & $\mathrm{XX}^{3}$ \\
\hline GAL & Compensation (Hi) & $\mathrm{XX}$ & $\mathrm{O}$ \\
\hline CALCA & Compensation (Hi) & $\mathrm{XX}$ & $\mathrm{O}$ \\
\hline HCRT & Compensation (Hi) & $\mathrm{XX}$ & $\mathrm{X}$ \\
\hline PNOC & Compensation (Hi) & $\#^{2}$ & $<>$ \\
\hline IL5 & Compensation (Hi) & $<>$ & $<>$ \\
\hline BCL2 & Compensation (Hy) & $<>$ & $<>$ \\
\hline IGFBP1 & Compensation (Hy) & $\mathrm{xx}^{3}$ & $<>$ \\
\hline IGFBP5 & Compensation (Hy) & $<>$ & $<>$ \\
\hline IL3 & Compensation (Hy) & $<>$ & $<>$ \\
\hline TRPV3 & Compensation (Hy) & $<>$ & $<>$ \\
\hline PRL7A3 & iCR exclusive (Hi) & $<>$ & $\mathrm{XX}^{3}$ \\
\hline PLCB1 & iCR exclusive (Hy) & $<>$ & $<>$ \\
\hline MAPK9 & iCR exclusive (Hy) & $<>$ & $<>$ \\
\hline ADCY1 & iCR exclusive (Hy) & $<>$ & $<>$ \\
\hline G6PC & iCR exclusive (Hy) & $<>$ & $<>$ \\
\hline CACNA1A & iCR exclusive (Hy) & $<>$ & $<>$ \\
\hline ESR1 & General interest & $\#^{1}$ & $\#^{1}$ \\
\hline ESR2 & General interest & $\#^{1}$ & $\mathrm{O}$ \\
\hline OPRM1 & General interest & $\mathrm{O}$ & $\#^{2}$ \\
\hline KISS1 & General interest & $<>$ & $\mathrm{O}$ \\
\hline TAC3 & General interest & $\#^{1}$ & $\mathrm{XX}^{3}$ \\
\hline
\end{tabular}

"XX" = qPCR and microarray mutually show 'compensation of OVX-induced effect by iCR'

"X" = qPCR and microarray mutually show 'exclusive effect of iCR'

"O" = qPCR and microarray show 'no relevant effect of iCR'

" $<>"=$ qPCR and microarray results are not consistent with each other "\#" = target genes, which do not show similar patterns at the two techniques but are worth to be discussed

1 compensation in qPCR, but no such effect found in the microarray

2 compensation in microarray, but in qPCR only a trend of an OVX-effect and a significant iCR-effect was found

3 compensation in qPCR, but in microarray only a trend was seen which did not meet the FC threshold criteria.

Publisher's Note Springer Nature remains neutral with regard to jurisdictional claims in published maps and institutional affiliations. 